\title{
Symmetry Energy of Dilute Warm Nuclear Matter
}

\author{
J. B. Natowitz, ${ }^{1}$ G. Röpke, ${ }^{2}$ S. Typel,${ }^{3,4}$ D. Blaschke,${ }^{5,6}$ A. Bonasera, ${ }^{1,7}$ K. Hagel, ${ }^{1}$ T. Klähn,,${ }^{5,8}$ S. Kowalski, ${ }^{1}$ L. Qin, ${ }^{1}$ \\ S. Shlomo, ${ }^{1}$ R. Wada, ${ }^{1}$ and H. H. Wolter ${ }^{9}$ \\ ${ }^{1}$ Cyclotron Institute, Texas A\&M University, College Station, Texas 77843-3366, USA \\ ${ }^{2}$ Institut für Physik, Universität Rostock, Universitätsplatz 3, D-18055 Rostock, Germany \\ ${ }^{3}$ Excellence Cluster Universe, Technische Universität München, Boltzmannstraße 2, D-85748 Garching, Germany \\ ${ }^{4}$ GSI Helmholtzzentrum für Schwerionenforschung GmbH, Theorie, Planckstraße 1, D-64291 Darmstadt, Germany \\ ${ }^{5}$ Instytut Fizyki Teoretycznej, Uniwersytet Wrocławski, plac Maksa Borna 9, 50-204 Wroctaw, Poland \\ ${ }^{6}$ Bogoliubov Laboratory for Theoretical Physics, JINR Dubna, Joliot-Curie street 6, 141980 Dubna, Russia \\ ${ }^{7}$ Laboratori Nazionali del Sud-INFN, via Santa Sofia 64, 95123 Catania, Italy \\ ${ }^{8}$ Theory Group, Physics Division, Building 203, Argonne National Laboratory, 9700 South Cass Avenue, Argonne, Illinois 60439, USA \\ ${ }^{9}$ Fakultät für Physik, Universität München, Am Coulombwall 1, D-85748 Garching, Germany
}

(Received 13 January 2010; published 17 May 2010)

\begin{abstract}
The symmetry energy of nuclear matter is a fundamental ingredient in the investigation of exotic nuclei, heavy-ion collisions, and astrophysical phenomena. New data from heavy-ion collisions can be used to extract the free symmetry energy and the internal symmetry energy at subsaturation densities and temperatures below $10 \mathrm{MeV}$. Conventional theoretical calculations of the symmetry energy based on mean-field approaches fail to give the correct low-temperature, low-density limit that is governed by correlations, in particular, by the appearance of bound states. A recently developed quantum-statistical approach that takes the formation of clusters into account predicts symmetry energies that are in very good agreement with the experimental data. A consistent description of the symmetry energy is given that joins the correct low-density limit with quasiparticle approaches valid near the saturation density.
\end{abstract}

DOI: 10.1103/PhysRevLett.104.202501

PACS numbers: 21.65.Ef, 25.70.Pq, 26.60.Kp

The symmetry energy [1] in the nuclear equation of state governs phenomena from the structure of exotic nuclei to astrophysical processes. The structure and the composition of neutron stars depend crucially on the density dependence of the symmetry energy [2]. As a general representation of the symmetry energy coefficient we use the definition

$$
E_{\mathrm{sym}}(n, T)=\frac{E(n, 1, T)+E(n,-1, T)}{2}-E(n, 0, T),
$$

where $E(n, \delta, T)$ is the energy per nucleon of nuclear matter with density $n$, temperature $T$, and asymmetry $\delta=$ $(N-Z) / A$ with $Z$ and $N$ the proton and neutron numbers, and $A=N+Z$. At low density the symmetry energy changes mainly because additional binding is gained in symmetric matter due to formation of clusters and pasta structures [3].

Our empirical knowledge of the symmetry energy near the saturation density $n_{0}$ is based primarily on the binding energies of nuclei. The Bethe-Weizsäcker mass formula leads to values of about $E_{\mathrm{sym}}\left(n_{0}, 0\right)=28-34 \mathrm{MeV}$ for the symmetry energy at zero temperature and saturation density $n_{0} \approx 0.16 \mathrm{fm}^{-3}$, if surface asymmetry effects are properly taken into account [4].

In contrast with the value of $E_{\mathrm{sym}}\left(n_{0}, 0\right)$, the variation of the symmetry energy with density and temperature is intensely debated. Many theoretical investigations have been performed to estimate the behavior of the symmetry energy as a function of $n$ and $T$. A recent review is given by $\mathrm{Li}$ et al. [5]; see also [6,7]. Typically, quasiparticle approaches such as the Skyrme Hartree-Fock and relativistic meanfield (RMF) models or Dirac-Brueckner Hartree-Fock (DBHF) calculations are used. In such calculations the symmetry energy tends to zero in the low-density limit for uniform matter. However, in accordance with the mass action law, cluster formation dominates the structure of low-density symmetric matter at low temperatures. Therefore, the symmetry energy in this low-temperature limit has to be equal to the binding energy per nucleon associated with the strong interaction of the most bound nuclear cluster. A single-nucleon quasiparticle approach cannot account for such structures. The correct low-density limit can be recovered only if the formation of clusters is properly taken into account, as has previously been shown in Ref. [8] in the context of a virial expansion valid at very low densities and in Ref. [9].

In this Letter we employ a quantum-statistical (QS) approach which includes cluster correlations in the medium. It interpolates between the exact low-density limit and the very successful RMF approaches near the saturation density. We show that this picture is in agreement with recent experimental findings on $E_{\text {sym }}$ at very low densities.

Suitable approaches to account for cluster formation are the nuclear statistical equilibrium (NSE) model [10], cluster-virial expansions [8], and generalized BethUhlenbeck approaches [11]. A thermal Green function 
approach that allows a generalization of the NSE model by introducing a quasiparticle description also for the bound states was already formulated some decades ago [12], but only recently analyzed with respect to the consequences for nuclear matter [13]. In this QS approach the cluster correlations are described in a generalized Beth-Uhlenbeck expansion. The advantage of this method is that the medium modifications of the clusters at finite density are taken into account. In Ref. [9] the thermodynamic properties of nuclear matter were derived using this approach. The formulation of Ref. [9] is valid in the density and temperature range where the formation of light clusters with $A \leq 4$ dominates and heavier clusters are not yet important. The method requires a sufficiently accurate model for the quasiparticle properties, for which we employ a RMF model with density dependent couplings [14] which gives a good description both of nuclear matter around normal density and of ground state properties of nuclei across the nuclear chart. In order to extend the applicability of this RMF model to very low densities, it has been generalized in Ref. [9] to account also for cluster formation and dissolution.

We note that at very low densities and temperatures below $T \simeq 1 \mathrm{MeV}$ new phases may occur. In fact, the formation of a solid phase using Overhauser orbitals including a triple point [15] or Bose-Einstein condensation [16] has been suggested. However, in this Letter we are concerned with experimental data which probe nuclear matter at considerably higher temperatures. The low- $T$ behavior is an interesting issue for future studies.

In the following we focus on finite temperatures and on the subsaturation region $n<n_{0}$. Experimental information is derived from heavy-ion collisions of charge asymmetric nuclei, where transient states of different density can be reached, depending on the incident energy and the centrality of the collision. In the Fermi energy domain symmetry energy effects have been investigated using judiciously chosen observables [5,17-20].

Recently, the experimental determination of the symmetry energy at very low densities produced in heavy-ion collisions of ${ }^{64} \mathrm{Zn}$ on ${ }^{92} \mathrm{Mo}$ and ${ }^{197} \mathrm{Au}$ at $35 \mathrm{MeV}$ per nucleon has been reported [21]. Results of this study are given in the first four columns of Table I. Note that, as a result of an energy recalibration and reevaluation of the particle yields in different velocity bins, these values are slightly different than those reported in Ref. [21]. The surface velocity $v_{\text {surf }}$, i.e., the velocity before the final Coulomb acceleration, was used as a measure of the time when the particles leave the source under different conditions of density and temperature. Only values of $v_{\text {surf }}<$ $4.5 \mathrm{~cm} / \mathrm{ns}$ are included here, since the system does not reach equilibrium for higher $v_{\text {surf }}$; see Table I of Ref. [21]. The yields of the light clusters $A \leq 4$ were determined as a function of $v_{\text {surf }}$. Temperatures were determined with the Albergo method [22] using a $\mathrm{H}-\mathrm{He}$ thermometer based on the double yield ratio of deuterons, tritons, ${ }^{3} \mathrm{He}$, and ${ }^{4} \mathrm{He}$, and are given in Table $I$ as the average for the two reactions.

The free neutron yield is obtained from the free proton yield and the yield ratio of ${ }^{3} \mathrm{H}$ to ${ }^{3} \mathrm{He}$. To determine the asymmetry parameter of the sources the total proton and neutron yields including those bound in clusters are used. The proton chemical potential is derived from the yield ratio of ${ }^{3} \mathrm{H}$ to ${ }^{4} \mathrm{He}$. The corresponding free proton and free neutron densities are calculated, and the total nucleon density is obtained by accounting also for the bound nucleons according to their respective yields [21]. The total nucleon densities are of the order of $1 / 100$ th to $1 / 20$ th of saturation density, as seen in Table I.

An isoscaling analysis [23] has been employed (as a function of $v_{\text {surf }}$ ) to determine the free symmetry energy $F_{\text {sym }}$ via the expression $\alpha=4 F_{\text {sym }} \Delta(Z / A)^{2} / T$. Here $\alpha$ is the isoscaling coefficient determined from yield ratios of $Z=1$ ejectiles of the two reactions and $\Delta(Z / A)^{2}$ is the difference of the squared asymmetries of the sources in the two reactions. With $\Delta(Z / A)^{2}$ and the temperature determined as above, the free symmetry energy is extracted.

Using the free symmetry energy derived in this way from the measured yields, the internal symmetry energy can be calculated if the symmetry entropy is known. The

TABLE I. Temperatures, densities, and free and internal symmetry energies for different values of the surface velocity as derived from heavy-ion collisions (columns 2-6), from the QS approach (columns 7-8), and self-consistently with clusters (columns 9-12); see text.

\begin{tabular}{|c|c|c|c|c|c|c|c|c|c|c|c|}
\hline $\begin{array}{c}V_{\text {surf }} \\
(\mathrm{cm} / \mathrm{ns})\end{array}$ & $\begin{array}{c}T \\
(\mathrm{MeV})\end{array}$ & $\begin{array}{c}n \\
\left(\mathrm{fm}^{-3}\right)\end{array}$ & $\begin{array}{c}F_{\text {sym }} \\
(\mathrm{MeV})\end{array}$ & $\begin{array}{l}S_{\mathrm{sym}}^{\mathrm{NSE}} \\
\left(k_{B}\right)\end{array}$ & $\begin{array}{c}E_{\text {sym }} \\
(\mathrm{MeV})\end{array}$ & $\begin{array}{c}F_{\text {sym }}^{\mathrm{QS}} \\
(\mathrm{MeV})\end{array}$ & $\begin{array}{c}E_{\mathrm{sym}}^{\mathrm{QS}} \\
(\mathrm{MeV})\end{array}$ & $\begin{array}{c}T^{\mathrm{SC}} \\
(\mathrm{MeV})\end{array}$ & $\begin{array}{c}n^{\mathrm{SC}} \\
\left(\mathrm{fm}^{-3}\right)\end{array}$ & $\begin{array}{c}F_{\mathrm{sym}}^{\mathrm{SC}} \\
(\mathrm{MeV})\end{array}$ & $\begin{array}{c}E_{\mathrm{sym}}^{\mathrm{SC}} \\
(\mathrm{MeV})\end{array}$ \\
\hline 0.75 & 3.31 & 0.00206 & 5.64 & 0.5513 & 7.465 & 6.607 & 8.011 & 3.26 & 0.00493 & 9.211 & 9.666 \\
\hline 1.25 & 3.32 & 0.00165 & 6.07 & 0.5923 & 8.036 & 6.087 & 7.502 & 3.45 & 0.00511 & 9.295 & 9.647 \\
\hline 1.75 & 3.61 & 0.00234 & 6.63 & 0.4137 & 8.124 & 6.877 & 7.896 & 3.54 & 0.00510 & 9.284 & 9.612 \\
\hline 2.25 & 4.15 & 0.00378 & 7.81 & 0.1557 & 8.456 & 8.184 & 8.305 & 3.66 & 0.00495 & 9.193 & 9.524 \\
\hline 2.75 & 4.71 & 0.00468 & 8.28 & -0.0162 & 8.204 & 8.967 & 8.321 & 4.02 & 0.00510 & 9.274 & 9.386 \\
\hline 3.25 & 5.27 & 0.00489 & 9.30 & -0.1358 & 8.584 & 9.395 & 7.785 & 4.65 & 0.00574 & 9.683 & 9.227 \\
\hline 3.75 & 6.24 & 0.00549 & 10.69 & -0.2936 & 8.858 & 10.729 & 7.623 & 5.75 & 0.00684 & 10.487 & 8.978 \\
\hline 4.25 & 7.54 & 0.00636 & 11.83 & -0.4197 & 8.665 & 11.397 & 7.807 & 7.46 & 0.00866 & 11.982 & 8.964 \\
\hline
\end{tabular}


values of the symmetry entropy $S_{\mathrm{sym}}^{\mathrm{NSE}}$ for given parameters of temperature and density within the NSE model are shown in Table I, column 5. They are calculated with the equivalent expression of Eq. (1) as the difference between the entropies of pure proton or neutron and symmetric nuclear matter. In contrast with the mixing entropy that leads to a larger entropy for uncorrelated symmetric matter in comparison with pure neutron matter, the formation of correlations, in particular, clusters, will reduce the entropy in symmetric matter; see also Fig. 9 of Ref. [9]. For parameter values for which the yields of free nucleons in symmetric matter are small, the symmetry entropy may become positive, as seen in Table I for low temperatures. The fraction of nucleons bound in clusters can decrease, e.g., due to increasing temperature or the dissolution of bound states at high densities due to the Pauli blocking. Then, the symmetric matter recovers its larger entropy so that the symmetry entropy becomes negative, as seen in Table I also in the QS and self-consistent (SC, see below) calculations.

The results obtained in this way for the internal symmetry energy $E_{\mathrm{sym}}=F_{\mathrm{sym}}+T S_{\mathrm{sym}}^{\mathrm{NSE}}$ are shown in Table I, column 6 . We note that in Ref. [21] the symmetry entropy was estimated using results of the virial expansion of Ref. [8] leading to different internal symmetry energies. However, this approximation is unreliable at the densities considered here.

In Table I, we also give results of the QS model [9] for the free and internal symmetry energies (columns 7 and 8) at given $T$ and $n$. In Fig. 1(a) the experimentally obtained free symmetry energy is compared to the results of the RMF calculation without clusters and the QS model with clusters [9]. There are large discrepancies between the measured values and the results of calculations in the mean-field approximation when cluster formation is neglected. On the other hand, the QS model results correspond nicely to the experimental data. In Fig. 1(b) we compare the internal symmetry energy derived from the experimental data with the RMF and QS results. Again, it
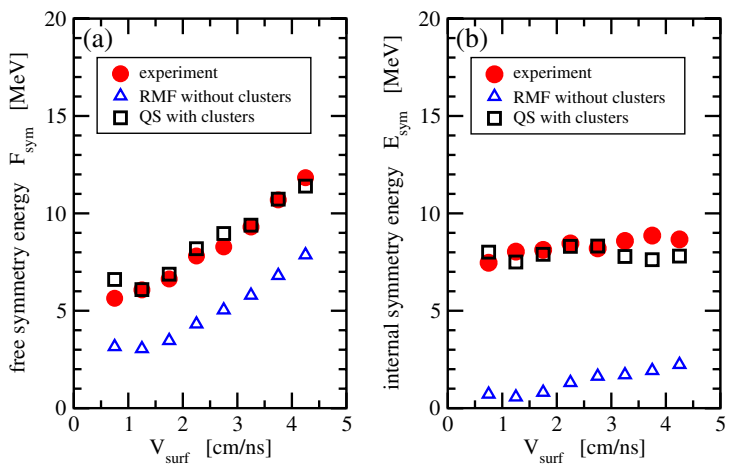

FIG. 1 (color online). Free (a) and internal (b) symmetry energy as a function of the surface velocity. Experimental results are compared with results of theoretical calculations neglecting cluster formation (RMF) and including cluster formation (QS). is clearly seen that the quasiparticle mean-field approach (RMF without clusters) disagrees strongly with the experimentally deduced symmetry energy while the QS approach gives a rather good agreement with the experimental data.

In Fig. 2 we present results for different approaches of extracting the internal symmetry energy and compare with the experimental values. In Fig. 2(a) we show theoretical results for $T$ at or close to zero. A widely used momentumdependent parametrization of the symmetry energy (MDI) at temperature $T=0 \mathrm{MeV}$ was given in Refs. [5,24] and is shown for different assumed values of the stiffness parameter $x$. For these parametrizations the symmetry energy vanishes in the low-density limit. We compare this to the $\mathrm{QS}$ result at $T=1 \mathrm{MeV}$ (at lower $T$ crystallization or Bose condensation may occur as discussed above). In this approach the symmetry energy is finite at low density. The $T=1 \mathrm{MeV}$ curve will also approach zero at extremely low densities of the order of $10^{-5} \mathrm{fm}^{-3}$ because the temperature is finite. The RMF, $T=0$ curve is discussed below. Also note that the underlying RMF model for the quasiparticle description with $n_{0}=0.149 \mathrm{fm}^{-3}$, $E_{\text {sym }}\left(n_{0}\right)=32.73 \mathrm{MeV}$ gives a reasonable behavior at high density similar to the MDI, $x=0$ parametrization. We thus see that our approach successfully interpolates between the clustering phenomena at low density and a realistic description around normal density.
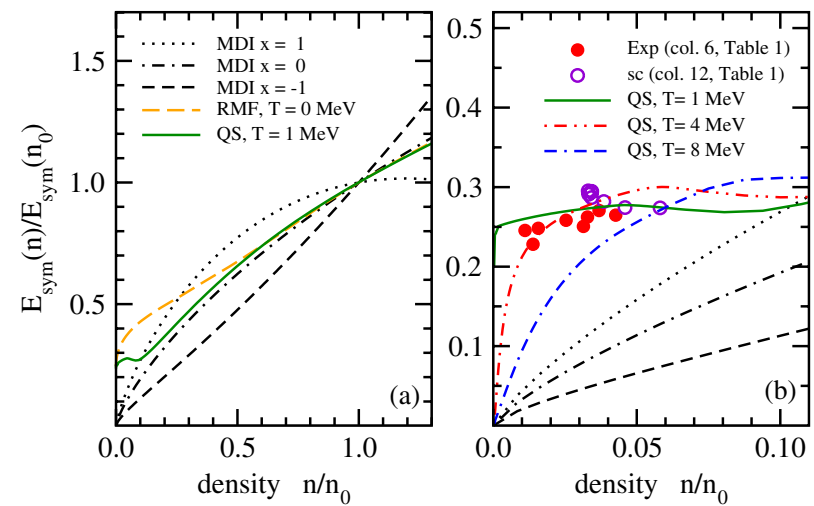

FIG. 2 (color online). Comparisons of the scaled internal symmetry energy $E_{\text {sym }}(n) / E_{\text {sym }}\left(n_{0}\right)$ as a function of the scaled total density $n / n_{0}$ for different approaches and the experiment. (a) The symmetry energies for the commonly used MDI parametrization of Chen et al. [24] for $T=0$ and different asy stiffnesses, controlled by the parameter $x$ [dotted, dot-dashed, and dashed (black) lines], for the QS model including light clusters for temperature $T=1 \mathrm{MeV}$ [solid (green) line], and for the RMF model at $T=0$ including heavy clusters [long-dashed (orange) line]. (b) The internal scaled symmetry energy in an expanded low-density region. Shown are again the MDI curves and the QS results for $T=1,4$, and $8 \mathrm{MeV}$ compared with the experimental data with the NSE entropy (solid circles) and the results of the self-consistent calculation (open circles) from Table I. 
In Fig. 2(b) we compare the theoretical results to the experimental ones, full (red) circles (Table I, column 6), in an expanded low-density region. In addition to the MDI parametrization, we show the QS results [9] for $T=1,4$, and $8 \mathrm{MeV}$, which are in the range of the temperatures in the experiment. The QS results including cluster formation agree well with the experimental data points, as seen in detail in Fig. 1. We conclude that medium-dependent cluster formation has to be considered in theoretical models to obtain the low-density dependence of the symmetry energy that is observed in experiments.

The temperatures and densities of columns 2 and 3 in Table I will be modified if medium effects on the light clusters are taken into account [25]. We have carried out a self-consistent determination of the temperatures $T^{\mathrm{SC}}$ and densities $n^{\mathrm{SC}}$ taking into account the medium-dependent quasiparticle energies as specified in Ref. [13] (columns 9 and 10 of Table I). Compared to the Albergo method results [21], the temperatures $T^{\mathrm{SC}}$ are about $10 \%$ lower. Significantly higher values are obtained for the inferred densities $n^{\mathrm{SC}}$ which are more sensitive to the inclusion of medium effects. We have also calculated the free and internal symmetry energies corresponding to these selfconsistent values of $T^{\mathrm{SC}}$ and $n^{\mathrm{SC}}$ according to Ref. [9] (columns 11 and 12 of Table I). These results are also shown in Fig. 2(b) as open (purple) circles. The resultant internal symmetry energies are 15\%-20\% higher than the QS model values for $T$ and $n$ given in columns 2 and 3 in Table I.

We have restricted our present work to that region of the phase diagram where heavier clusters with $A>4$ are not relevant. The generalization of the given approach to account for clusters of arbitrary size would lead to an improvement in the low-density, low-temperature region when nuclear statistical equilibrium is assumed. Alternatively, one can introduce the formation of heavier nuclei in the presence of a nucleon and cluster gas, cf. Refs. [26,27].

The simplest approach to model the formation of heavy clusters is to perform inhomogeneous mean-field calculations in the Thomas-Fermi approximation assuming spherical Wigner-Seitz cells. In Fig. 2(a) preliminary results for the zero-temperature symmetry energy of such a calculation is shown by the long-dashed line using the same RMF parametrization as for the QS approach introduced above; for details see Ref. [28]. The symmetry energy in this model approaches a finite value at zero density in contrast with the behavior of the MDI parametrizations and conventional single-nucleon quasiparticle descriptions.

In conclusion, we have shown that a quantum-statistical model of nuclear matter, which includes the formation of clusters at densities below nuclear saturation, describes quite well the low-density symmetry energy which was extracted from the analysis of heavy-ion collisions. Within such a theoretical approach the composition and the thermodynamic quantities of nuclear matter can be modeled in a large region of densities, temperatures, and asymmetries that are required, e.g., in supernova simulations.

This research was supported by the DFG cluster of excellence "Origin and Structure of the Universe," by CompStar, a Research Networking Programme of the European Science Foundation, by U.S. Department of Energy Contract No. DE-AC02-06CH11357 (T. K.) and Grant No. DE-FG03-93ER40773 (Texas A\&M), and by Robert A. Welch Foundation Grant No. A0330 (J.B.N.). D. B. acknowledges support from the Polish Ministry for Research and Higher Education, Grant No. N N 202231837 and from the Russian Fund for Basic Research, Grant No. 08-02-01003-a.

[1] We use the historical term "symmetry energy" although the community should invoke renaming it "asymmetry energy" as it characterizes the dependence of the nuclear binding energy on the asymmetry $\delta=(N-Z) / A$.

[2] J. M. Lattimer and M. Prakash, Phys. Rep. 442, 109 (2007).

[3] G. Watanabe et al., Phys. Rev. Lett. 103, 121101 (2009).

[4] P. Danielewicz, Nucl. Phys. A727, 233 (2003).

[5] B. A. Li et al., Phys. Rep. 464, 113 (2008).

[6] C. Fuchs and H. H. Wolter, Eur. Phys. J. A 30, 5 (2006).

[7] T. Klähn et al., Phys. Rev. C 74, 035802 (2006).

[8] C. J. Horowitz and A. Schwenk, Nucl. Phys. A776, 55 (2006).

[9] S. Typel, G. Röpke, T. Klähn, D. Blaschke, and H. H. Wolter, Phys. Rev. C 81, 015803 (2010).

[10] J. P. Bondorf et al., Phys. Rep. 257, 133 (1995).

[11] M. Schmidt, G. Röpke, and H. Schulz, Ann. Phys. (N.Y.) 202, 57 (1990).

[12] G. Röpke et al., Nucl. Phys. A379, 536 (1982); A424, 594 (1984).

[13] G. Röpke, Phys. Rev. C 79, 014002 (2009).

[14] S. Typel, Phys. Rev. C 71, 064301 (2005).

[15] H. R. Jaqaman, Phys. Rev. C 38, 1418 (1988).

[16] H. Takemoto et al., Phys. Rev. C 69, 035802 (2004).

[17] V. Baran et al., Phys. Rep. 410, 335 (2005).

[18] M. B. Tsang et al., Phys. Rev. Lett. 102, 122701 (2009).

[19] S. Wuenschel et al., Phys. Rev. C 79, 061602(R) (2009).

[20] C. Sfienti et al., Phys. Rev. Lett. 102, 152701 (2009).

[21] S. Kowalski et al., Phys. Rev. C 75, 014601 (2007).

[22] S. Albergo et al., Nuovo Cimento Soc. Ital. Fis. 89A, 1 (1985).

[23] M. B. Tsang et al., Phys. Rev. Lett. 86, 5023 (2001).

[24] L. W. Chen et al., Phys. Rev. Lett. 94, 032701 (2005); Phys. Rev. C 76, 054316 (2007).

[25] S. Shlomo et al., Phys. Rev. C 79, 034604 (2009).

[26] J. M. Lattimer et al., Nucl. Phys. A535, 331 (1991).

[27] H. Shen et al., Nucl. Phys. A637, 435 (1998).

[28] S. Typel et al. (to be published). 